\title{
Rogowski Coil for Current Measurement in a Cryogenic Environment
}

\author{
Tao $\mathrm{Ma}^{1,2}$, Shaotao Dai ${ }^{1,2}$, Jingye Zhang ${ }^{1,2}$ and Lianqi Zhao ${ }^{3}$ \\ ${ }^{1}$ Institute of Electrical Engineering, Chinese Academy of Sciences, Beijing, 100190 China, \\ matao@mail.iee.ac.cn \\ ${ }^{2}$ Key Laboratory of Applied Superconductivity, Chinese Academy of Sciences, Beijing, 100190 China, \\ ${ }^{3}$ Jiangsu Zhongtian Technology Co., Ltd., Nantong, Jiangsu, 226463 China.
}

\begin{abstract}
A Rogowski coil based sensor for current measurement in a cryogenic environment and results of its application for paralleled high temperature superconducting (HTS) coil current sharing are presented. The current sensor consists of a Rogowski coil and an integrator, where the coil output voltage is proportional to the derivative of primary current and the integrator transfers the differentiation to normal state. The Rogowski coil has promising applicability at cryogenic circumstance because its body is made of low temperature materials. The integrator ensures a large bandwidth with feasible magnitude, which is vital for dynamic current measurement during the quench of the HTS coil. The proposed current sensor is used for current sharing measurement of two paralleled Bi2223 HTS coils, and the experimental results show that the measurement precision is better than $0.5 \%$.
\end{abstract}

Keywords: Rogowski coil, integrator, current measurement, high temperature superconducting coil, cryogenic.

\section{INTRODUCTION}

$\mathrm{T}$ HE PROMISE of low-cost high temperature superconducting (HTS) conductors coupled with reasonably priced refrigeration systems has encouraged application of this technology to a variety of magnets and power equipments [1]. Unfortunately, as the conductor ampacity and withstand voltage are limited, it is crucial to connecting the conductors in series/parallel configurations to meet the voltage and current requirements. Therefore, the voltage and current sharing of used conductors become a vital part during design and assembling, since unbalanced current or voltage may be a threat to safety operation of the conductor, or even cause damage.

As the voltage can be lead out from cryogenic to room temperature without losses, conventional voltage measurement skills are available to voltage sharing testing. On the other hand, conventional current measurement devices cannot work at cryogenic temperature $(<100 \mathrm{~K})$, copper current leads are commonly inserted so that conventional current measurement devices at room temperature can be used. However, the impedance of inserted current leads may seriously affect the current distribution of parallel conductors since the impedance of an HTS conductor is relatively small. Thus, it is better for us to measure the current in liquid nitrogen without long current leads.

Current sensing techniques are mainly based upon Ohm's law of resistance, the Faraday effect, magnetic field sensors and Faraday's law of induction [2]. The current sensors based on Ohm's law of resistance, like shunt resistor and trace resistance sensing, often provide the advantage of lower costs compared with other sensing techniques, and have the reputation of being reliable due to their simple working principle. However, a significant drawback for the application of such kind of current sensor is the unavoidable electrical connection between the current to be measured and the sensing circuit, which is undesirable at deep low temperature.

Current sensors based on Faraday effect, like fiber-optic current sensor, are especially useful in high voltage systems because of the inherent electrical isolation, and in systems with high electromagnetic-inference levels, but not suitable for low temperature application as the fiber-optic deforms at extremely low temperature, which deteriorates the accuracy.

Magnetic field sensors provide an attractive alternative basis for both alternating and direct current sensing. These magnetic field current sensors based on the Hall-effect, fluxgate principle and magneto resistance effect are widely used and accepted nowadays in conventional current measurement. However, the most serious limitation is the degaussing cycle required after an over-current incident, and the distinct temperature related drift of the output voltage, which is not applicable for cryogenic temperature situation. Superconducting quantum interference device (SQUID) is extremely useful for magnetic field measurement in a cryogenic environment, but it is difficult to use at a high voltage situation [3].

Current transformer based on Faraday's law of induction is the most widely used current sensing device [4]. However, the magnetic core material may cause the HTS tape to quench, and the size is relatively large in order to measure large current. The Rogowski coil is another kind of current sensor based on Faraday's law of induction as current transformer. The Rogowski coil usually uses non-magnetic core, and the sensing probe structure is very simple, which are very important for low temperature application [5]. A distinct feature of the Rogowski coil is that it does not exhibit saturation, and is inherently linear $[6,7]$. It has been widely used in impulse [8], large-magnitude [9], and some high-frequency [10] current measurement.

Nevertheless, the thermal expansion may change the cross- 
sectional area of the coil, which is extremely apparent in a liquid nitrogen environment and affects the accuracy. On the other hand, compared with the current transformer, the sensitivity of the Rogowski coil is small. More turns on the coil or large integrator gain could increase the sensitivity. However, more turns increase the self-inductance and distributed capacitance, whereas a higher integrator gain requires an amplifier with large gain-bandwidth product, which reduces the circuit bandwidth [11]. A bandwidth of $2.5 \mathrm{kHz}$ is enough for the measurement of power grid current harmonics [12], and we could have a trade-off between the bandwidth and the number of turns [13]. When plunged at cryogenic temperature, the coil resistance, distributed capacitance, and self-inductance are all changing, which changes the coil output voltage.

This paper solves the problem of alternating dynamic current measurement at cryogenic temperature using the Rogowski coil. Considering the measurement requirement of the developed superconducting equipment, a Rogowski coil with a bandwidth of $1.67 \sim 2000 \mathrm{~Hz}$ is developed, and the thermal expansion and essential impedance change of the coil from room temperature to cryogenic temperature are discussed. The cross-sectional area change may extremely affect the measurement accuracy, thus the materials for both coil core and turns are carefully selected as Stycast 2850 FT and copper, since they have relatively small and similar thermal expansion coefficient and low-temperature characteristics. Consequently, the current sharing of paralleled HTS coils is measured using those calibrated low temperature Rogowski coil current sensors. The experimental results show that the developed Rogowski coil has an accuracy around $0.5 \%$ at low temperature with the frequency of $1.67 \mathrm{~Hz} \sim 2000 \mathrm{~Hz}$ and current range of $100 \mathrm{Arms} \sim 2000 \mathrm{~A}$, and we could acquire exactly the current distribution of paralleled HTS coils.

\section{PRINCIPLE OF THE ROGOWSKI COIL CURRENT SENSOR}

\subsection{Fundamentals}

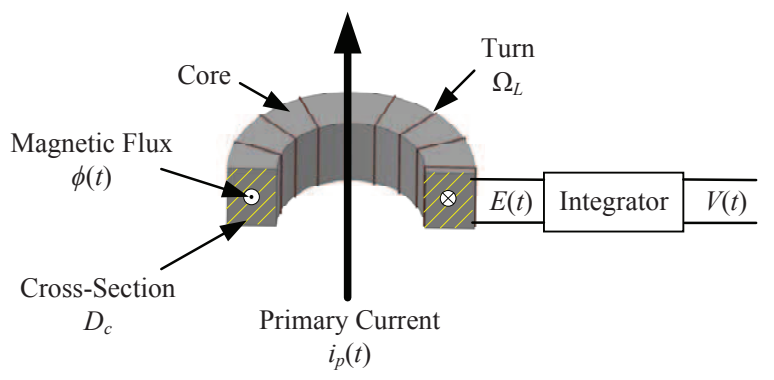

Fig. 1: Rogowski coil with an integrator

The basic layout of a current sensor using the Rogowski coil is shown in Fig. 1, where the Rogowski coil usually comprises an annular core with a turn of uniform density and area [14]. According to Faraday's law of induction, an AC (alternating current) primary current $i_{p}(t)$ generates an AC mag- netic flux $\phi(t)$ in the cross-section area $D_{c}$ surrounded by the turns. Consequently, the AC magnetic flux $\phi(t)$ induces the electromotive force $E(t)$. It is not difficult to find that the electromotive force $E(t)$ is proportional to the differential of primary current $i_{p}(t)$. Therefore, an integrator is necessary for the sensor.

The working principle starts with Ampere's law that defines the path integral of the magnetic flux density $B$ inside the coil

$$
\oint_{\Omega_{L}} \vec{B} \cdot d \vec{l}=\mu_{0} i_{p}(t)
$$

The primary conductor current $i_{p}(t)$ flows through the area enclosed by turn $\Omega_{L}$. To simplify the theoretical analysis, we assume that:

A0. The cross section diameter of the Rogowski coil is much smaller than its radius.

A1. The current $i_{p}(t)$ is centered inside the Rogowski coil.

A2. The cross-section of the Rogowski coil is the same for the full length of the core.

A3. The turn around the core is uniform.

\subsection{Error source caused by thermal expansion}

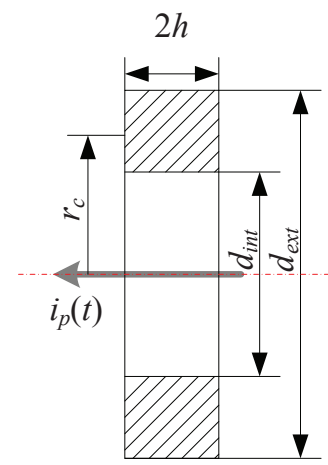

Fig. 2: Rectangular section

A Rogowski coil with rectangular section is shown in Fig. 2, where $d_{i n t}$ and $d_{\text {ext }}$ are the internal and external diameters, respectively, and $2 h$ denotes the height of the coil. With the above assumption A0-A1 and (1), the magnetic flux density $B$ at one spot at distance $r_{C}$ from the coil becomes

$$
B=\frac{\mu_{0} i_{p}(t)}{2 \pi r_{C}}
$$

where $r_{C}$ is the distance from the axis of primary current lead to the specific spot on the coil, and $\mu_{0}=4 \pi \times 10^{-7} \mathrm{H} / \mathrm{m}$ is space permeability. By applying the Faraday's law of induction and (2), a measured current $i_{p}(t)$ induces an AC magnetic flux $\phi_{j}(t)$ in one turn,

$$
\phi_{j}(t)=\frac{\mu_{0} i_{p}(t)}{2 \pi} \int_{-h}^{h} \int_{d_{\text {int }} / 2}^{d_{e x t} / 2} \frac{1}{x} d x d y=\frac{\mu_{0} i_{p}(t) h}{\pi} \ln \frac{d_{\text {ext }}}{d_{\text {int }}}
$$


According to A2, if the cross-section of the Rogowski coil is the same for the full length of the core, the electromotive force in the Rogowski coil is

$$
E(t)=\sum_{j=1}^{N}-\frac{d \phi_{j}(t)}{d t}=-\frac{\mu_{0} N h}{\pi} \ln \frac{d_{\text {ext }}}{d_{\text {int }}} \frac{d i_{p}(t)}{d t}
$$

where $N$ is the number of turns. From the above equation (4), the electromotive force $E(t)$ is proportional to the derivative of the primary current $i_{p}(t)$. Therefore, the transfer function between the primary current and the electromotive force becomes

$$
E(s)=-s N \phi_{j}(s)=-s \cdot \frac{\mu_{0} N h}{\pi} \cdot \ln \frac{d_{\text {ext }}}{d_{\text {int }}} \cdot i_{p}(s)
$$

The above (4) is the foundation for using the Rogowski coil for current measurement. It is based on the assumption A2, that the cross-section of the Rogowski coil is the same for the full length of the core, which is reasonable in common application. However, the shape and circuit parameters of the coil vary when soaked into liquid nitrogen. Although the variation of dimensions will lead particularly to variation of crosssection area $D_{c}$, some relation between the dimensions will remain the same. Nevertheless, it is important for us to investigate the effect of such low temperature around $77 \mathrm{~K}$. The impact of geometry change on magnetic flux $\phi_{j}(t)$ is:

$$
\begin{aligned}
\frac{\partial \phi_{j}(t)}{\partial h} & =\frac{\mu_{0} i_{p}(t)}{\pi} \ln \frac{d_{\text {ext }}}{d_{\text {int }}} \\
\frac{\partial \phi_{j}(t)}{\partial R} & =\frac{\mu_{0} i_{p}(t) h}{\pi} \frac{1}{d_{\text {ext }}} \\
\frac{\partial \phi_{j}(t)}{\partial r} & =-\frac{\mu_{0} i_{p}(t) h}{\pi} \frac{1}{d_{i n t}}
\end{aligned}
$$

Thus,

$$
\partial \phi_{j}(t)=\frac{\mu_{0} i_{p}(t)}{\pi}\left[\ln \frac{d_{e x t}}{d_{i n t}} \cdot \partial h+h\left(\frac{\partial d_{e x t}}{d_{e x t}}-\frac{\partial d_{i n t}}{d_{i n t}}\right)\right]
$$

Therefore, the impact of geometry change on the electromotive force becomes

$$
\partial E(s)=-\frac{\mu_{0} N s i_{p}(s)}{\pi}\left[\ln \frac{d_{e x t}}{d_{i n t}} \cdot \partial h+h\left(\frac{\partial d_{e x t}}{d_{e x t}}-\frac{\partial d_{i n t}}{d_{i n t}}\right)\right]
$$

Remark 1. From (8), the output of a Rogowski coil with rectangular section is proportional changes to the axial and radial variation. As the value of $2 h$ decreases, the signal gain decreases proportionally, namely, a variation by height results in the same percentage of measurement error. However, the variation of height does not affect the phase. It is not difficult to find that the error caused by coil external diameter $d_{\text {ext }}$ is similar to that of coil height $2 \mathrm{~h}$. However, compared with external diameter $d_{\text {ext }}$, the internal diameter $d_{\text {int }}$ enlarges, and the signal gain increases.

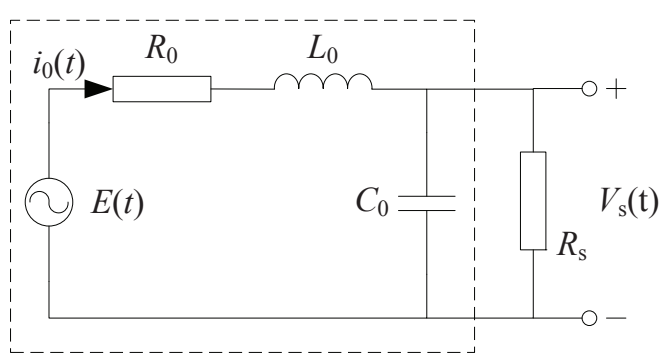

Fig. 3: Lumped-element model of a Rogowski coil

Fig. 3 shows the lumped-element model of a Rogowski coil, where $R_{0}, L_{0}, C_{0}$ are the lumped resistance, inductance and capacitance of the coil, respectively, and $R_{S}$ denotes the terminal matching resistance of the coil. $E(t)$ and $V_{s}(t)$ are the induced electromotive force and coil output voltage, respectively, and $i_{0}(t)$ is the loop current of the coil.

Therefore, the transfer function $G_{0}(s)$ of the Rogowski coil with terminal matching resistor is

$$
G_{0}(s)=\frac{V_{s}(s)}{E(s)}=\frac{R_{s}}{s^{2} R_{s} L_{0} C_{0}+s\left(L_{0}+R_{0} R_{s} C_{0}\right)+R_{0}+R_{s}}
$$

Since the impedance of a Rogowski coil changes at low temperature, we get the impact from (9)

$$
\begin{aligned}
\frac{\partial G_{0}(s)}{\partial R_{0}} & =-\frac{R_{s}\left(1+s R_{s} C_{0}\right)}{\Delta^{2}} \\
\frac{\partial G_{0}(s)}{\partial L_{0}} & =-\frac{s R_{S}\left(1+s R_{s} C_{0}\right)}{\Delta^{2}} \\
\frac{\partial G_{0}(s)}{\partial C_{0}} & =-\frac{s R_{s}{ }^{2}\left(R_{0}+s L_{0}\right)}{\Delta^{2}}
\end{aligned}
$$

where $\Delta \triangleq s^{2} R_{s} L_{0} C_{0}+s\left(L_{0}+R_{0} R_{s} C_{0}\right)+R_{0}+R_{s}$.

Remark 2. (10a)-(10c) present the influence of self inductance $L_{0}$, resistance $R_{0}$, and distributed capacitance $C_{0}$, respectively. Comparing those three equations with (6a)-(6c), they do not affect apparently the signal magnitude and phase as the coil dimension does. What needs a specification is that temperature obviously affects the resistivity, and the resistance $R_{0}$ has more serious impact than $L_{0}$ and $C_{0}$.

\section{SELECTION OF THE MATERIAL AND INTEGRATOR}

Achieving ideal performance in a practical Rogowski coil demands considerable care in its design. According to the aforementioned impact of low temperature on a Rogowski coil, the material for such coil should be carefully selected.

\subsection{Material for core and turns}

The core of the Rogowski coil can be made of any nonmagnetic material [15]. Some magnetic materials are also used to enhance the induced voltage. From Remark 1, in order to maintain the coil performance, it is vital that the crosssectional area remains essentially constant when the coil is immersed in liquid nitrogen. 
MEASUREMENT SCIENCE REVIEW, Volume 15, No. 2, 2015

Table 1: Linear thermal contraction $[16,17,18]$

\begin{tabular}{lc}
\hline Material & $\Delta l / l_{293}\left(10^{-4}\right) \mathbf{2 9 3} \mathbf{K}$ to $\mathbf{8 0 ~ K}$ \\
\hline $\mathrm{Al}$ & 39.0 \\
$\mathrm{Cu}$ & 30.2 \\
Pyrex glass & 5.4 \\
$\mathrm{Al}_{2} \mathrm{O}_{3}$ ceramic & 15 \\
Stycast 2850 FT & $47.8(77 \mathrm{~K})$ \\
$\mathrm{G} 10(\perp)$ & 64 \\
$\mathrm{G} 10(\|)$ & 21 \\
\hline
\end{tabular}

However, almost all materials experience a change in physical dimension when cooled to cryogenic temperatures, which is usually referred to as thermal contraction. The contraction in volume can have profound effect on the design of devices operating at low temperature, largely because the thermal contractions of different materials, as well as their mechanical properties, vary considerably. Unfortunately, engineering devices operating in cryogenic systems are composed of a number of different materials, therefore, the interaction and matching of used materials becomes the major concern.

Integral thermal contraction of some materials is presented in Table 1. The coil conductor needs to be non-ferromagnetic material, thus iron and stainless steel are not suitable. Besides, the magnet wire on the market is usually made of copper, thus the copper magnet wire other than aluminum is used for the coil turns. From Table 1, the thermal contraction rate of the $\mathrm{Al}_{2} \mathrm{O}_{3}$ ceramic and pyrex glass material is small, but they are more complicated to process and more brittle at low temperatures. From Table 1, the thermal contraction ratio of G10 type glass fiber reinforced epoxy composite materials is significantly anisotropic, which is inappropriate to the uniform requirement of the coil. Commonly used cryogenic temperature epoxy Stycast 2850 FT material with catalyst $24 \mathrm{LV}$ has a thermal contraction rate of $47.8 \times 10^{-4}$ at $77 \mathrm{~K}$, and it is good of strength at cryogenic temperature and easy for plastics molding. Although its thermal contraction rate is larger than copper magnetic wire, the Stycast 2850 FT is used to build the coil core for its mechanical strength at cryogenic temperature. In order to decrease the linear expansion rate, some fiberglass is added to the coil core, and the fiberglass is carefully knitted to decrease the anisotropic.

\subsection{Circuit of integrator}

The processing circuit is composed of matching terminating resistor, amplifier, integrator, and a low-pass filter.

The overall parameters of the designed Rogowski coil are shown in Table 2, where $M \triangleq \frac{\mu_{0} N h}{\pi} \cdot \ln \frac{d_{\text {ext }}}{d_{\text {int }}}$ is the mutual inductance coefficient. The number of turns is decided according to the measurement dimension requirement and the frequency response from (5) and (9). To ensure that the coil has an appropriate damping, overshoot, and rise time, the terminating resistance should be [19]

$$
R_{s}=\frac{L_{0}}{\sqrt{2 L_{0} C_{0}-R_{0}^{2} C_{0}^{2}}}
$$

Table 2: Rogowski coil parameters

\begin{tabular}{lc}
\hline Parameter & Value $(\mathbf{3 0 0} \mathbf{~ K})$ \\
\hline Inner diameter $d_{\text {int }}(\mathrm{mm})$ & 25 \\
External diameter $d_{\text {ext }}(\mathrm{mm})$ & 45 \\
Height $2 h(\mathrm{~mm})$ & 15 \\
Coil wire diameter $d_{w}(\mathrm{~mm})$ & 0.23 \\
Number of turns $N$ & 335 \\
Resistance $R_{0}(\Omega)$ & 5.79 \\
Self inductance $L_{0}(\mu \mathrm{H})$ & 66.3 \\
Mutual inductance $M(\mu \mathrm{H})$ & 1.1815 \\
Distributed capacitance $C_{0}(\mathrm{pF})$ & 129 \\
\hline
\end{tabular}

Namely, $R_{s}=507 \Omega$ for the designed rectangle cross section Rogowski coil.

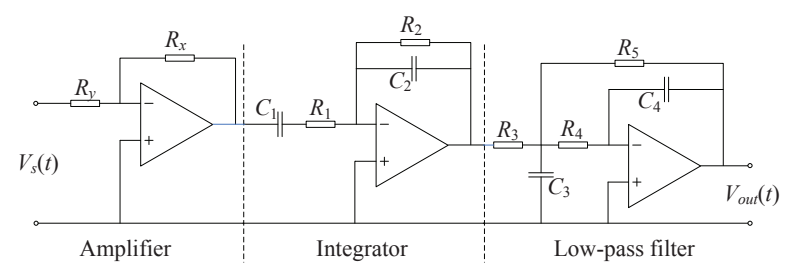

Fig. 4: Circuit of integrator

Fig. 4 shows the circuit of integrator, which includes an amplifier, an integrator, and a low-pass filter. The amplifier is used to amplify the weak Rogowski coil output signal, and meanwhile, to increase the loading capacity. Since the output of the Rogowski coil characterizes the differential of the current to be determined, an integrator is applied to reconstruct the current. A large negative feedback resistance $R_{2}$ is used to prevent the saturation caused by long term zero drift of the operational amplifier in the integrator. However, such kind of negative feedback integrator amplifies low frequency and direct current. Therefore, a large capacitance $C_{1}$ is allocated before the integrator to suppress the unwanted direct or extremely low frequency interference signals. On the other hand, to eliminate the high frequency interference signal coupled in the signal channel, a low-pass filter is employed. The used low-pass filter is a second order Butterworth filter with cut-off frequency $f_{c}=2000 \mathrm{~Hz}$.

Consequently, we get the transfer function of the integrator from Fig. 4:

$$
\begin{aligned}
& G_{\text {int }}(s)=-\frac{R_{x}}{R_{y}} \cdot \frac{s R_{2} C_{1}}{\left(1+s R_{1} C_{1}\right)\left(1+s R_{2} C_{2}\right)} \\
& \cdot \frac{R_{5} / R_{3}}{1+s C_{4} R_{4} R_{5}\left(\frac{1}{R_{3}}+\frac{1}{R_{4}}+\frac{1}{R_{5}}\right)+s^{2} C_{3} C_{4} R_{4} R_{5}}
\end{aligned}
$$

Thus, the transfer function of the designed current sensor is:

$$
G(s)=\frac{V_{\text {out }}(s)}{E(s)}=\frac{V_{\text {out }}(s)}{V_{s}(s)} \cdot \frac{V_{s}(s)}{E(s)}=G_{\text {int }}(s) \cdot G_{0}(s)
$$


where $G_{0}(s)$ is denoted by (9), and $G_{\text {int }}(s)$ is the transfer function of the integrator in (12).

According to reference [19], large negative feedback resistor $R_{2}$ broadens the bandwidth, but too large a resistor makes the amplifier work under strong negative feedback and unstable. Therefore, the value of negative feedback resistor $R_{2}$ in the integrator is chosen to be $1 \mathrm{M} \Omega$. Taking the signal gain, bandwidth, and sensitivity into consideration, we choose $R_{1}=10 \mathrm{k} \Omega, C_{2}=0.5 \mu \mathrm{F} . C_{1}$ is used to suppress the unwanted very low frequency interference signal, which should be much larger than $C_{2}$. Thus, $C_{1}$ is chosen to be $10 \mu \mathrm{F}$.

As was mentioned before, the cut-off frequency of low-pass filter is $2000 \mathrm{~Hz}$. Based on the Butterworth filter designing rules, denote $C_{3}=5 \mathrm{nF}, C_{4}=470 \mathrm{pF}, R_{3}=20 \mathrm{k} \Omega, R_{4}=40 \mathrm{k} \Omega$ and $R_{5}=66 \mathrm{k} \Omega$.

By adjusting the value of $R_{x}$, we set the proportional coefficient of this Rogowski coil current transducer to be 400 . Namely, the rated output of integrator is $5 \mathrm{~V}$, while the primary conductor current is $2000 \mathrm{~A}$. First, let $R_{y}=1 \mathrm{k} \Omega$, then $R_{x}$ is approximately $3.3 \mathrm{k} \Omega$.

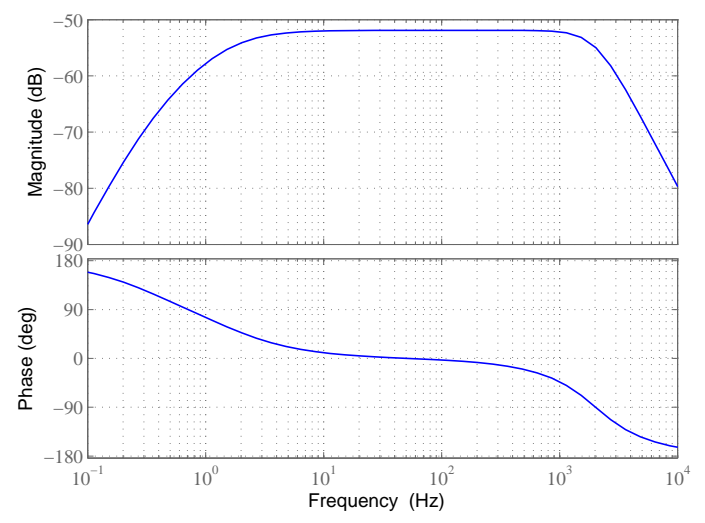

Fig. 5: Bode diagram of current transducer

Fig. 5 shows the bode diagram of the Rogowski coil current transducer based on (9) and (12). The low and high cut-off frequency at $3 \mathrm{~dB}$ is $1.67 \mathrm{~Hz}$ and $2000 \mathrm{~Hz}$, respectively. The purpose of this design is to measure the current change of the HTS tape for power system application, and the current does not change as fast as the pulsed one. Therefore, a high cut-off frequency of $2000 \mathrm{~Hz}$ is introduced to suppress the high frequency interference. On the other hand, the low-frequency or DC component of the input current is not linearly amplified as the middle-frequency band is, and the low-frequency interference may be amplified. Thus, a low cut-off frequency about $1.67 \mathrm{~Hz}$ is selected.

Remark 3. Within the above settings, the values of the designed Rogowski coil at $300 \mathrm{~K}$ are shown in Table 2. When soaked in liquid nitrogen at $77 \mathrm{~K}$, the coil shrinks about $0.5 \%$, namely, the height $2 \mathrm{~h}$ becomes about $14.925 \mathrm{~mm}$ and the external diameter becomes about $44.775 \mathrm{~mm}$. However, the inner diameter enlarges about $0.5 \%$. Therefore, according to
(7), the impact of geometry change on the electromotive force is $-2.2 \%$, which affects the final output with the same proportion.

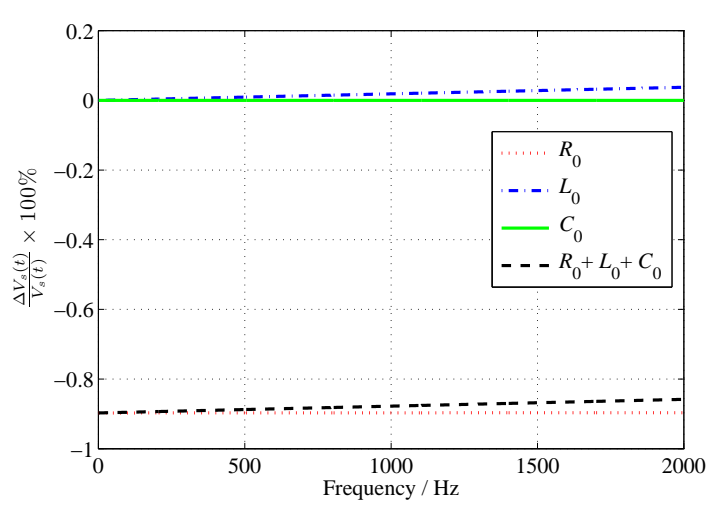

(a)

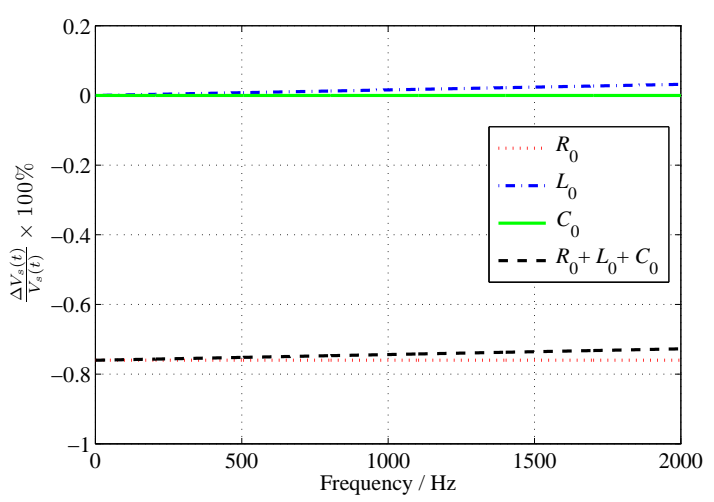

(b)

Fig. 6: Variation of $V_{s}(t)$ caused by circuit parameters: (a) $R_{S}=$ $507 \Omega$, (b) $R_{S}=598.6 \Omega$

Fig. 6 shows the variation of $\frac{\Delta V_{s}(t)}{V_{s}(t)} \times 100 \%$ caused by circuit parameters from (9). The conductor resistivity decreases and the permittivity and permeability of used materials changes at low temperature. However, no permittivity and permeability value of the used coil core material epoxy Stycast 2850 FT is known, neither at room nor low temperature. It is believed that the variation of permittivity value and dimension affects the capacitance, and permeability value and dimension affects the inductance. According to our test, the circuit parameters in Fig. 3 vary from $R_{0}=5.79 \Omega$, $L_{0}=66.3 \mu \mathrm{H}$ and $C_{0}=129 p F$ to $R_{0}=1.23 \Omega, L_{0}=81.7 \mu \mathrm{H}$ and $C_{0}=114 \mathrm{pF}$.

Fig. 6(a) shows the variation expressed as a percentage under the condition that the terminating resistance in (11) is calculated within parameters given at room temperature, i.e. $R_{s}=507 \Omega$. It is not difficult to see that the variation of resistance $R_{0}$ has a more serious impact as was described in Remark 2 , and the inductance has a positive deviation while the capacitance and resistance gets a negative one. Besides, the influence of self inductance and distributed capacitance increases as the frequency enlarges from $1.67 \mathrm{~Hz}$ to $2000 \mathrm{~Hz}$. 
The capacitive reactance of $X_{C_{0}}=\frac{1}{2 \pi f_{c} C_{0}}$ varies from $617 \mathrm{k} \Omega$ to $698 \mathrm{k} \Omega$, and the inductive reactance of $X_{L_{0}}=2 \pi f_{c} L_{0}$ varies from $0.8327 \Omega$ to $1.0262 \Omega$, respectively at $2000 \mathrm{~Hz}$. Thus, from (9) we could see that the coil output voltage $V_{s}(t)$ varies from $0.9871 E(t)$ to $0.9956 E(t)$. Namely, the overall influence of self inductance $L_{0}$, resistance $R_{0}$, and distributed capacitance $C_{0}$ is $-0.86 \%$, which affects the final output with the same proportion as the geometry change does.

Fig. 6(b) shows the variation when the terminal matching resistance in (11) is calculated within parameters given at low temperature $(77 \mathrm{~K})$, i.e. $R_{S}=598.6 \Omega$. Compared with Fig. 6(a), the influence of the circuit parameters variation is slightly smaller. This is due to the increase in the terminal matching resistance $R_{s}$, and the impedance variation of the coil is relatively smaller than that within $R_{S}=507 \Omega$. Similarly, the overall influence of self inductance $L_{0}$, resistance $R_{0}$, and distributed capacitance $C_{0}$ is $-0.73 \%$, is smaller than the $-0.86 \%$ in Fig. 6(a).

\section{MEASUREMENT OF HTS COILS CURRENT SHARING}

Since the current distribution of parallelized HTS coil is crucial for the safety operation of superconducting equipments, we have to measure the current sharing without current lead at low temperature in order to overcome the impact of additional copper current lead impedance. First, we have to calibrate the designed Rogowski coil at low temperature. Thus, the coils are used for the measurement of current sharing in steady and dynamic states.

\subsection{Calibration of Rogowski coil in liquid nitrogen}

After the design and testing of the Rogowski coil at room temperature, we calibrate the current sensor in room temperature. Thus, the relationship between the coil output $V_{s}(t)$ and the output of integrator $V_{\text {out }}(t)$ can be obtained exactly, and denote $V_{\text {out }}(t)=F\left(V_{s}(t)\right)$. Since the integrator and the matching resistor $R_{s}$ are not changed when the coil is plugged into cryogenic temperature, the function $F(\cdot)$ does not change. However, since the relationship between the primary current $i_{p}(t)$ and the coil output $V_{s}(t)$ changes, we have to further calibrate it in liquid nitrogen environment according to the aforementioned impact of low temperature on the coil output [20, 21].

The developed Rogowski coil is calibrated in liquid nitrogen at $77 \mathrm{~K}$, and several Hall-effect current sensors with different measuring range and a bandwidth from DC-10 kHz are used as reference. Fig. 7 shows the measured current error versus reference current at various frequencies of $1.67 \mathrm{~Hz}$, $10 \mathrm{~Hz}, 20 \mathrm{~Hz}, 50 \mathrm{~Hz}, 100 \mathrm{~Hz}, 200 \mathrm{~Hz}, 500 \mathrm{~Hz}, 1000 \mathrm{~Hz}$, and $2000 \mathrm{~Hz}$. A repeat experiment has produced similar results that the measured current error at the range of $0 \sim 10 \%$ rated current is larger than $1.0 \%$, and the linearity of the sensor output is better than $0.5 \%$ at the range of $10 \% \sim 100 \%$ rated current. Therefore, this coil is not appropriate for the measurement of current lower than 100 Arms. Furthermore, more than 10 times of repeat experimental results show that the measurement error difference at different frequencies is less than

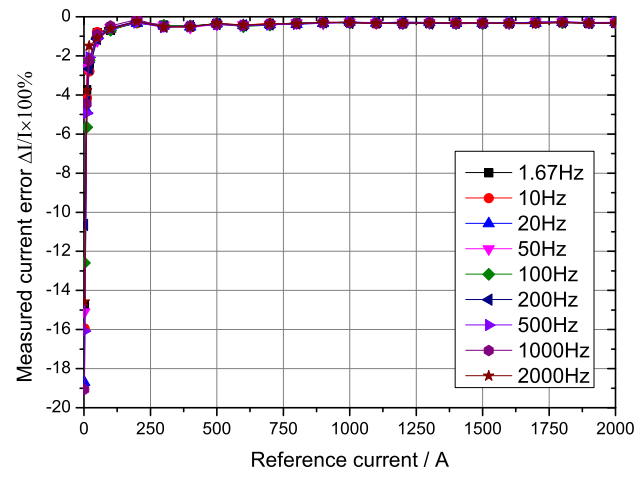

Fig. 7: Measured current error versus reference current at various frequencies

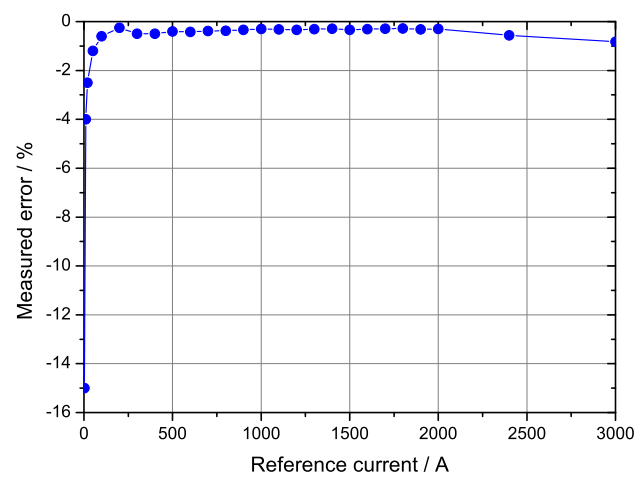

Fig. 8: Measured current error versus reference current at $50 \mathrm{~Hz}$

$0.1 \%$. However, the measurement accuracy reduces to about $0.5 \%$ when the current frequency approaches to the low-cutoff frequency $1.67 \mathrm{~Hz}$ and high-cutoff frequency $2000 \mathrm{~Hz}$.

Fig. 8 shows the measured current carried out by the designed Rogowski coil after calibration in liquid nitrogen at $50 \mathrm{~Hz}$. It is not difficult to see that the measured current error at the range of $10 \% \sim 90 \%$ rated current is less than $0.5 \%$, which is consistent with the results in Fig. 7. However, when the reference current is less than $10 \%$ of the rated current, the percentage of the measured error increases. The measured error is about $-15 \%$ when the primary current of the coil is 2 Arms. On the other hand, when the reference current is larger than $100 \%$, the measured error increases too, where the measured error is about $-0.8 \%$ at $150 \%$ rated current. It is due to the magnetic-frequency response becoming nonlinear, as shown in Fig. 5.

\subsection{HTS coils current sharing}

With the calibrated low temperature Rogowski coils, we could measure the current sharing of HTS coils at both steady and dynamic states. The used coils are made of Bi2223 from Sumitomo with critical current around $170 \mathrm{~A}$.

Fig. 9a presents the structure of the connection, which shows that the current lead of HTS coil B is longer than that 


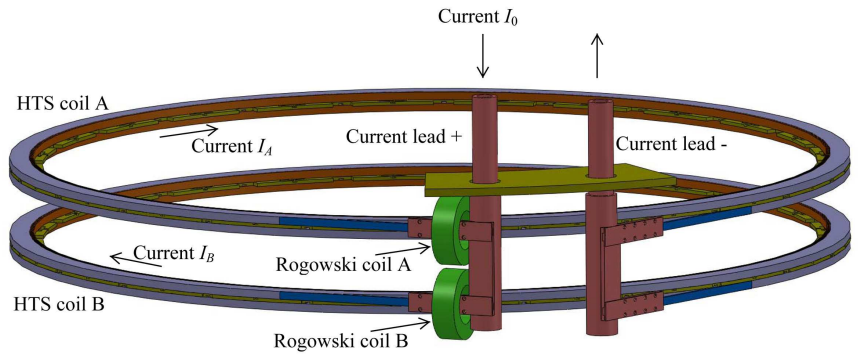

(a)

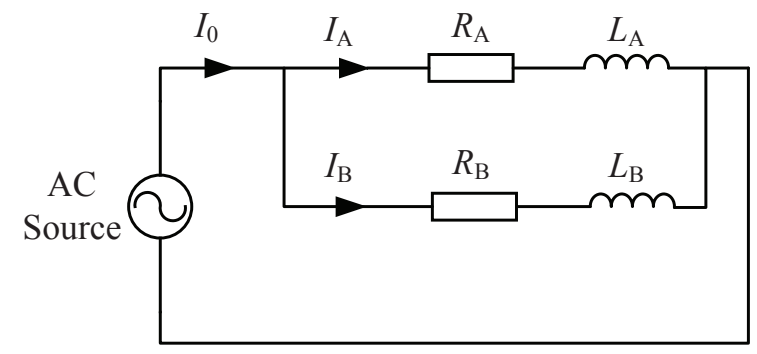

(b)

Fig. 9: Testing of paralleled HTS coils current sharing

of HTS coil A. As the resistance of superconducting coil is far lower than the current lead made of copper, the current sharing of the two coils is determined by the coil inductance and the current lead resistance. From Fig. 9b, $R_{A}$ denotes the resistance of branch A including the current lead resistance, the contact resistance and the superconducting coil AC resistance, and $R_{B}$ denotes the resistance of branch $\mathrm{B}$. Besides, $L_{A}$ and $L_{B}$ denotes the inductance of branch $\mathrm{A}$ and $\mathrm{B}$, respectively. In this experiment, $L_{A}=L_{B}=129 \mu \mathrm{H}$, but it is difficult to make a distinction between $R_{A}$ and $R_{B}$. Nevertheless, the resistance is far lower than the reactance of the coil. However, since the contact resistance and the coil AC resistance of both coils are almost the same, the longer current lead of branch B makes $R_{A} \neq R_{B}$. According to the testing results, $R_{A}$ and $R_{B}$ are less than $50 \mu \Omega$ at steady state. Therefore, circuit impedance of each branch is determined mainly by coil reactance at steady state.

Fig. 10 shows the current of each branch in Fig. 9 at steady state, where $I_{A}, I_{B}, I_{0}$ denote the measured current of branch $\mathrm{A}$, branch $\mathrm{B}$, and the reference main circuit, respectively, and $I_{A}+I_{B}$ indicates the main current calculated from the branches. $I_{A}, I_{B}$ are acquired from the calibrated Rogowski coils. It is not difficult to find that the calculated current is similar to the reference one with a bias less than $0.5 \%$, namely, the designed Rogowski coils work well in a cryogenic environment.

Fig. 11 shows the current of each branch in Fig. 9 at quench. Large current exceeding the critical current makes both of the paralleled superconducting coils quench quickly, and their resistance rise fast. Therefore, the circuit impedance of each branch is determined mainly by coil resistance other than coil inductance. Consequently, compared with Fig. 10, the difference between $I_{A}$ and $I_{B}$ is much smaller. Still, we can see

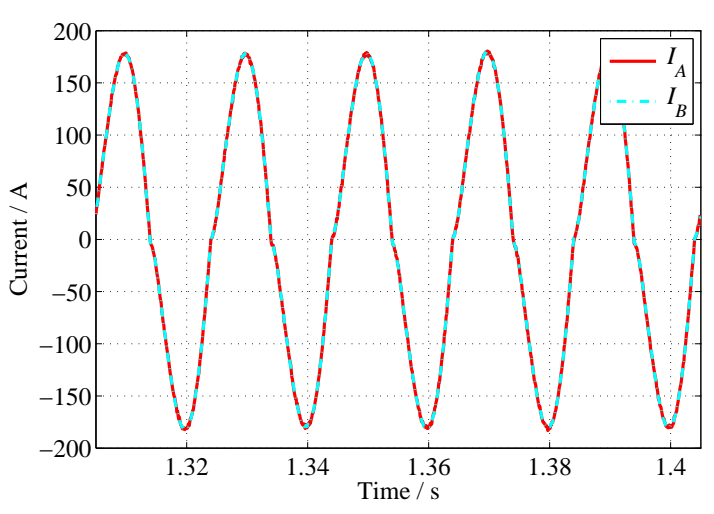

(a)

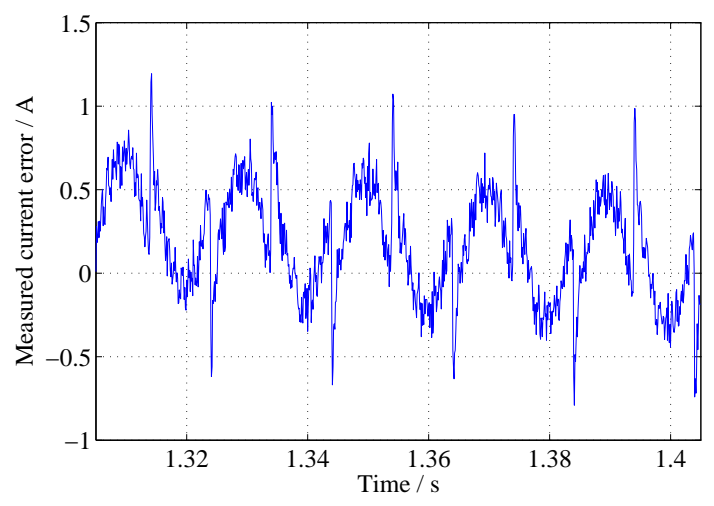

(b)

Fig. 10: Current sharing at steady state: (a) Current of each branch, (b) Measured current error $\Delta I=I_{0}-\left(I_{A}+I_{B}\right)$

that the calculated current $I_{A}+I_{B}$ is almost the same as the reference $I_{0}$ with a bias no more than $0.5 \%$.

\section{CONCLUSION}

Several Rogowski coils are designed for cryogenic environment application. The impact of low temperature on the coil is carefully discussed, which shows that the thermal contraction changes the coil output proportionally. The precision of the Rogowski coil for current measurement can be better than $0.5 \%$ after calibration. Using the designed low temperature Rogowski coil for current sharing of HTS coils, we could see that the resistance of current lead affects the current distribution for each branch at steady state. As the resistance of HTS coil rises after quench, the current on each branch varies gradually.

\section{ACKNOWLEDGEMENT}

This research was supported by the National Science Foundation of China (61403361, 51477168), the Ministry of Sciences and Technology of China (2012AA050403), and Jiangsu Province of China(BY2012245). 


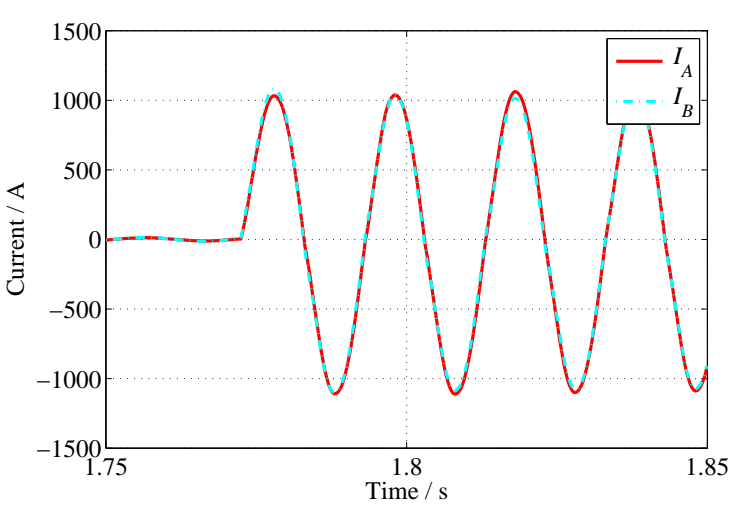

(a)

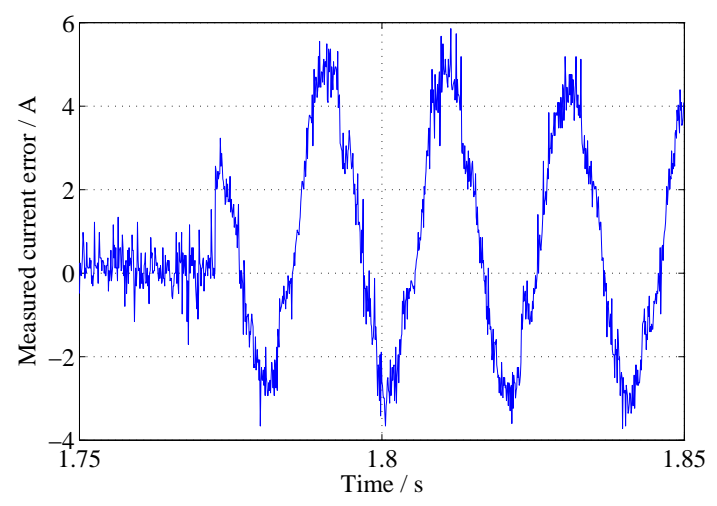

(b)

Fig. 11: Current sharing at quench: (a) Current of each branch, (b) Measured current error $\Delta I=I_{0}-\left(I_{A}+I_{B}\right)$

\section{REFERENCES}

[1] Kalsi, S.S. (2011). Applications of High Temperature Superconductors to Electric Power Equipment. John Wiley \& Sons.

[2] Ziegler, S., Woodward, R.C., Iu, H.H., et al. (2009). Current sensing techniques: A review. IEEE Sensors Journal, 9 (4), 354-376.

[3] Kiwa, T., Miyazaki, S., Saari, M.M., et al. (2014). Visualization of ion transportation in an electrolyte using an HTS-SQUID gradiometer. Physica C: Superconductivity, 504, 84-87.

[4] Slawomir, T. (2007). Induction coil sensors: A review. Measurement Science and Technology, 18, R31-R46.

[5] Gonzalez-Parada, A., Espinosa-Loza, F.J., Bosch-Tous, R. (2012). Inductive method for the determination of the critical current Ic of HTS stators in axial flux configuration. IEEE Transactions on Applied Superconductivity, 22 (3), 9500104.

[6] Cataliotti, A., Cara, D.D., Emanuel, A.E., et al. (2011). Characterization and error compensation of a Rogowski coil in the presence of harmonics. IEEE Transations on Instrumentation and Measurement, 60 (4), 1175-1181.

[7] Djokic, B.V., Ramboz, J.D., Destefan, D.E. (2011). To what extent can the current amplitude linearity of Ro- gowski coils be verified? IEEE Transations on Instrumentation and Measurement, 60 (7), 2409-2414.

[8] Metwally, I.A. (2013). Performance improvement of slow-wave Rogowski coils for high impulse current measurement. IEEE Sensors Journal, 13 (2), 538-547.

[9] Metwally, I.A. (2013). Coaxial-cable wound Rogowski coils for measuring large-magnitude short-duration current pulses. IEEE Transations on Instrumentation and Measurement, 62 (1), 119-128.

[10] Shafiq, M., Kutt, L., Lehtonen, M., et al. (2013). Parametrs identification an modeling of high-frequency current transducer for partial discharge measurements. IEEE Sensors Journal, 13 (3), 1081-1091.

[11] Chiampi, M., Crotti, G., Morando, A. (2011). Evaluation of flexible Rogowski coil performances in power frequency applications. IEEE Transations on Instrumentation and Measurement, 60 (3), 854-862.

[12] Chinese Standard. (1993). Quality of electric energy supply: Harmonics in public supply network. GB/T 14549-93. Beijing.

[13] Isa, M., Elkalashy, N.I., Lehtonen, M., et al. (2012). Multi-end correlation-based PD location technique for medium voltage covered-conductor lines. IEEE Transations on Dielectrics and Electrical Insulation, 19 (3), 936-946.

[14] Marracci, M., Tellini, B., Zappacosta, C., et al. (2011). Critical parameters for mutual inductance between Rogowski coil and primary conductor. IEEE Transations on Instrumentation and Measurement, 60 (2), 625-632.

[15] Abdi-Jalebi, E., McMahon, R. (2007). Highperformance low-cost Rogowski transducers and accompanying circuitry. IEEE Transations on Instrumentation and Measurement, 56 (3), 753-759.

[16] Guy, K.W., Philip, J.M. (2006). Experimental Techniques in Low-Temperature Physics: Fourth Edition. Oxford University Press.

[17] Wang, Y.S. (2011). Fundamental of Superconducting Power Technology. Beijing, China: Scientific Press.

[18] Steve, D. (1990). Report on thermal contraction of some materials including Stycast 2850 FT. Fermilab Materials Development Lab.

[19] Liu, Y., Lin, F.C., Zhang, Q., et al. (2011). Design and construction of a Rogowski coil for measuring wide pulsed current. IEEE Sensors Journal, 11 (1), 123-130.

[20] Draxler, K., Styblikova, R., Hlavacek, J., et al. (2011). Calibration of Rogowski coils with an integrator at high currents. IEEE Transations on Instrumentation and Measurement, 60 (7), 2434-2438.

[21] Hemmati, E., Shahrtash, S.M. (2013). Digital compensation of Rogowski coil's output voltage. IEEE Transations on Instrumentation and Measurement, 62, 71-82.

Received November 04, 2014. Accepted April 30, 2015. 